PROCEEDINGS OF THE

AMERICAN MATHEMATICAL SOCIETY

Volume 125, Number 3, March 1997, Pages 775-778

S 0002-9939(97)03705-

\title{
THE FIRST INSTABILITY INTERVAL FOR HILL EQUATIONS WITH SYMMETRIC SINGLE WELL POTENTIALS
}

\author{
MIN-JEI HUANG
}

(Communicated by Palle E. T. Jorgensen)

\begin{abstract}
For Hill equations with symmetric single well (or symmetric single barrier) potentials, the first instability interval is absent when and only when the potential is constant.
\end{abstract}

\section{INTRODUCTION}

The Hill equation in its standard form is

$$
-y^{\prime \prime}(x)+q(x) y(x)=\lambda y(x),
$$

where $\lambda$ is a parameter, and $q(x)$ is a real-valued, continuous and periodic function of period $a$. Let $y_{1}$ and $y_{2}$ be the linearly independent solutions of (1) which satisfy the initial conditions

$$
y_{1}(0, \lambda)=y_{2}^{\prime}(0, \lambda)=1, \quad y_{1}^{\prime}(0, \lambda)=y_{2}(0, \lambda)=0 .
$$

The discriminant of (1) is defined by

$$
D(\lambda)=y_{1}(a, \lambda)+y_{2}^{\prime}(a, \lambda) .
$$

It is known (see e.g. $[4,9]$ ) that $\lambda_{0}, \lambda_{1}, \lambda_{2}, \ldots$, the zeros of $D(\lambda)-2$, are the eigenvalues of (1) subject to the periodic boundary conditions $y(0)=y(a)$ and $y^{\prime}(0)=y^{\prime}(a)$, while $\mu_{0}, \mu_{1}, \mu_{2}, \ldots$, the zeros of $D(\lambda)+2$, are the eigenvalues of (1) subject to the semi-periodic boundary conditions $y(0)=-y(a)$ and $y^{\prime}(0)=-y^{\prime}(a)$. These eigenvalues are interlaced in the following way:

$$
\lambda_{0}<\mu_{0} \leq \mu_{1}<\lambda_{1} \leq \lambda_{2}<\mu_{2} \leq \mu_{3}<\lambda_{3} \leq \lambda_{4}<\cdots .
$$

For $\lambda \in\left(\lambda_{2 m}, \mu_{2 m}\right) \cup\left(\mu_{2 m+1}, \lambda_{2 m+1}\right)$, all solutions of (1) are bounded in $(-\infty, \infty)$. For $\lambda \in\left(-\infty, \lambda_{0}\right) \cup\left(\mu_{2 m}, \mu_{2 m+1}\right) \cup\left(\lambda_{2 m+1}, \lambda_{2 m+2}\right)$, all nontrivial solutions of (1) are unbounded in $(-\infty, \infty)$. The intervals $\left(\mu_{2 m}, \mu_{2 m+1}\right)$ and $\left(\lambda_{2 m+1}, \lambda_{2 m+2}\right)$ are called the $(2 m+1)$ th and $(2 m+2)$ th instability intervals, while $\left(-\infty, \lambda_{0}\right)$ is called the zeroth instability interval.

In 1946, G. Borg [3] proved an inverse theorem for a Hill's equation:

Theorem 1.1. The potential $q(x)$ has period a/2 if and only if all the odd instability intervals are absent, or, equivalently, all zeros of $D(\lambda)+2$ are double zeros.

Received by the editors July 10, 1995.

1991 Mathematics Subject Classification. Primary 34B30, 34L15.

Key words and phrases. Hill equation, eigenvalue, instability interval, symmetric single well potential.

(C) 1997 American Mathematical Society 
From this remarkable theorem he drew:

Theorem 1.2. The potential $q(x)$ is a constant if and only if all the instability intervals, except the zero-th, are absent.

For alternative proofs of these results, see the papers of Hochstadt $[5,6,7]$ and Ungar [10].

In this note, we are concerned with the first instability interval for symmetric single well potentials and symmetric single barrier potentials. By a symmetric single well potential on $[0, a]$ we mean a continuous function $q(x)$ on $[0, a]$ which is symmetric about $x=a / 2$ and nonincreasing on $[0, a / 2]$. The negative of a symmetric single well potential is called a symmetric single barrier potential. The purpose of this note is to prove the following

Theorem 1.3. Let $q(x)$ be a symmetric single well (or symmetric single barrier) potential on $[0, a]$. Then $q(x)$ is a constant if and only if the first instability interval is absent.

\section{Proof of the Result}

We denote by $\Lambda_{n}$ and $\nu_{n}(n=0,1,2, \ldots)$, respectively, the eigenvalues of (1) subject to the Dirichlet boundary condition $y(0)=y(a)=0$ and the Neumann boundary condition $y^{\prime}(0)=y^{\prime}(a)=0$. Then it is known [4] that $\nu_{0} \leq \lambda_{0}$ and for $n=0,1,2, \ldots$,

$$
\mu_{2 n} \leq \Lambda_{2 n} \leq \mu_{2 n+1}, \quad \lambda_{2 n+1} \leq \Lambda_{2 n+1} \leq \lambda_{2 n+2},
$$

and

$$
\mu_{2 n} \leq \nu_{2 n+1} \leq \mu_{2 n+1}, \quad \lambda_{2 n+1} \leq \nu_{2 n+2} \leq \lambda_{2 n+2} .
$$

Let $\psi_{0}(x)$ be a normalized eigenfunction corresponding to $\lambda_{0}$, so that $\psi_{0}(x)$ is periodic with period $a$ and $\int_{0}^{a}\left|\psi_{0}(x)\right|^{2} d x=1$. We first note:

Lemma 2.1. If $q(x)$ is symmetric about $x=a / 2$, then $\lambda_{0}=\nu_{0}$ and $\psi_{0}(x)$ is a Neumann eigenfunction corresponding to $\nu_{0}$.

Proof. Since $q(x)$ is periodic with period $a$ and symmetric about $x=a / 2, q(x)$ is an even function. It follows that if $y(x)$ is a solution of (1), then so also is $y(-x)$. In particular, $\psi_{0}(x)$ and $\psi_{0}(-x)$ are solutions of

$$
-y^{\prime \prime}(x)+q(x) y(x)=\lambda_{0} y(x)
$$

which satisfy the periodic boundary conditions. Since $\lambda_{0}$ is a simple eigenvalue, there is a constant $c$ such that $\psi_{0}(-x)=c \psi_{0}(x)$. But $\psi_{0}(x)$ has no zeros in $[0, a]$ (see [4]), so $\psi_{0}(-x)=\psi_{0}(x)$, hence $\psi_{0}^{\prime}(0)=\psi_{0}^{\prime}(a)=0$. This shows that $\psi_{0}(x)$ is a Neumann eigenfunction corresponding to $\lambda_{0}$. Since $\lambda_{0}<\mu_{0} \leq \nu_{1}$ by (2) and (4), we conclude that $\lambda_{0}=\nu_{0}$.

We note that $\psi_{0}(x)$ can be chosen so as to be positive for all $x$. Also, if $\Psi_{0}(x)$ is a normalized Dirichlet eigenfunction corresponding to $\Lambda_{0}$, then $\Psi_{0}(x)$ can be chosen so as to be positive for $x \in(0, a)$.

Lemma 2.2. If $q(x)$ is symmetric about $x=a / 2$, then the function $\Psi_{0}(x) / \psi_{0}(x)$ is increasing on $[0, a / 2]$ and decreasing on $[a / 2, a]$. 
Proof. Define $W(x)=\psi_{0}(x) \Psi_{0}^{\prime}(x)-\psi_{0}^{\prime}(x) \Psi_{0}(x)$ so that

$$
\left[\frac{\Psi_{0}(x)}{\psi_{0}(x)}\right]^{\prime}=\frac{W(x)}{\psi_{0}^{2}(x)} .
$$

By the differential equation and the fact that $\lambda_{0}<\Lambda_{0}$, we have

$$
\begin{aligned}
W^{\prime}(x) & =\psi_{0}(x) \Psi_{0}^{\prime \prime}(x)-\psi_{0}^{\prime \prime}(x) \Psi_{0}(x) \\
& =\left(\lambda_{0}-\Lambda_{0}\right) \psi_{0}(x) \Psi_{0}(x)<0
\end{aligned}
$$

for $x \in(0, a)$. Since $q(x)$ is symmetric about $x=a / 2$, the same is true for $\psi_{0}(x)$ and $\Psi_{0}(x)$, so $\psi_{0}^{\prime}\left(\frac{a}{2}\right)=\Psi_{0}^{\prime}\left(\frac{a}{2}\right)=0$. Hence $W\left(\frac{a}{2}\right)=0$. It follows that $W(x)>0$ for $x \in(0, a / 2)$ while $W(x)<0$ for $x \in(a / 2, a)$. This together with (5) proves the lemma.

Remark. If $q(x)$ is symmetric about $x=a / 2$, then so also is $\Psi_{0}(x) / \psi_{0}(x)$. Since $\psi_{0}$ and $\Psi_{0}$ are normalized, it follows from Lemma 2.2 that there are points $x_{ \pm}$with

$$
0<x_{-}<\frac{a}{2}<x_{+}<a, \quad x_{-}+x_{+}=a
$$

such that

$$
\Psi_{0}(x)<\psi_{0}(x) \quad \text { on }\left(0, x_{-}\right) \cup\left(x_{+}, a\right), \quad \Psi_{0}(x)>\psi_{0}(x) \quad \text { on }\left(x_{-}, x_{+}\right) .
$$

Using ideas of Lavine [8], we now prove:

Lemma 2.3. If $q(x)$ is a symmetric single well potential on $[0, a]$, then $\Lambda_{0}-\lambda_{0} \leq$ $\pi^{2} / a^{2}$. If $q(x)$ is a symmetric single barrier potential on $[0, a]$, then $\Lambda_{0}-\lambda_{0} \geq \pi^{2} / a^{2}$. In either case, the equality holds if and only if $q(x)$ is a constant.

Proof. Consider the one-parameter family of potentials $q(x, t)=t q(x), t \in \mathbf{R}$. We denote by $\lambda_{0}(t)$ and $\psi_{0}(x, t)$ the first eigenvalue and normalized eigenfunction of the corresponding Hill equation with periodic boundary conditions. Similarly, we use $\Lambda_{0}(t)$ and $\Psi_{0}(x, t)$ for Dirichlet boundary condition. As we know, $\lambda_{0}(0)=0$ and $\Lambda_{0}(0)=\pi^{2} / a^{2}$. By the standard formula, we have

$$
\frac{d}{d t} \lambda_{0}(t)=\int_{0}^{a} q(x) \psi_{0}^{2}(x, t) d x, \quad \frac{d}{d t} \Lambda_{0}(t)=\int_{0}^{a} q(x) \Psi_{0}^{2}(x, t) d x .
$$

So, if $\Gamma(t)=\Lambda_{0}(t)-\lambda_{0}(t)$, then

$$
\begin{aligned}
\frac{d}{d t} \Gamma(t)= & \int_{0}^{a} q(x)\left[\Psi_{0}^{2}(x, t)-\psi_{0}^{2}(x, t)\right] d x \\
= & \int_{\left[0, x_{-}(t)\right] \cup\left[x_{+}(t), a\right]} q(x)\left[\Psi_{0}^{2}(x, t)-\psi_{0}^{2}(x, t)\right] d x \\
& \quad+\int_{x_{-}(t)}^{x_{+}(t)} q(x)\left[\Psi_{0}^{2}(x, t)-\psi_{0}^{2}(x, t)\right] d x
\end{aligned}
$$

Now, suppose $q(x)$ is a symmetric single well potential. Then, by the preceding remark, $q(x) \geq q\left(x_{-}(t)\right)=q\left(x_{+}(t)\right)$ and $\Psi_{0}^{2}(x, t)-\psi_{0}^{2}(x, t)<0$ on $\left(0, x_{-}(t)\right) \cup$ $\left(x_{+}(t), a\right)$, while $q(x) \leq q\left(x_{-}(t)\right)$ and $\Psi_{0}^{2}(x, t)-\psi_{0}^{2}(x, t)>0$ on $\left(x_{-}(t), x_{+}(t)\right)$. Thus,

$$
\frac{d}{d t} \Gamma(t) \leq q\left(x_{-}(t)\right) \int_{0}^{a}\left[\Psi_{0}^{2}(x, t)-\psi_{0}^{2}(x, t)\right] d x=0 .
$$


This implies that $\Gamma(1) \leq \Gamma(0)$, i.e., $\Lambda_{0}-\lambda_{0} \leq \pi^{2} / a^{2}$, and the equality holds only if $q(x)$ is a constant. The result for symmetric single barrier potentials follows by a similar argument.

We are now ready to prove Theorem 1.3.

Proof. The only if part is obvious. To prove the if part, we consider only the case where $q(x)$ is a symmetric single well potential since the proof for barrier potentials is similar. Suppose that the first instability interval for $q(x)$ is absent. Then we have $\mu_{0}=\Lambda_{0}=\nu_{1}=\mu_{1}$ by (3) and (4). Thus, by Lemmas 2.1 and $2.3, \nu_{1}-\nu_{0} \leq \pi^{2} / a^{2}$. On the other hand, it was shown in [8] (see also $[1,2]$ ) that among symmetric single well potentials the gap $\nu_{1}-\nu_{0}$ is minimized at constant $q$, i.e., $\nu_{1}-\nu_{0} \geq \pi^{2} / a^{2}$. It follows that $q(x)$ is a constant. This completes the proof.

\section{REFERENCES}

1. M. Ashbaugh and R. Benguria, Optimal lower bounds for eigenvalue gaps for Schrödinger operators with symmetric single-well potentials and related results, Maximum Principles and Eigenvalue Problems in Partial Differential Equations (P. W. Schaefer, ed.), Pitman Res. Notes Math. Ser., vol. 175, Longman Sci. Tech., Harlow, 1988, pp. 134-145. MR 90c:35157

2. __ Optimal lower bound for the gap between the first two eigenvalues of one-dimensional Schrödinger operators with symmetric single-well potentials, Proc. Amer. Math. Soc. 105 (1989), 419-424. MR 89f:81028

3. G. Borg, Eine Umkehrung der Sturm-Liouvilleschen Eigenwertaufgabe. Bestimmung der Differentialgleichung durch die Eigenwerte, Acta Math. 78 (1946), 1-96. MR 7:382d

4. M. S. P. Eastham, The Spectral Theory of Periodic Differential Equations, Scottish Academic Press, Edinburgh and London, 1973.

5. H. Hochstadt, Functiontheoretic properties of the discriminant of Hill's equation, Math. Zeit. 82 (1963), 237-242. MR 27:5955

6. $\quad$ On the determination of a Hill's equation from its spectrum I, II, Arch. Rational Mech. Anal. 19 (1965), 353-362; 23 (1966), 237-238. MR 31:6019; MR 34:411

7. 373-374. MR 56:3404

8. R. Lavine, The eigenvalue gap for one-dimensional convex potentials, Proc. Amer. Math. Soc. 121 (1994), 815-821. MR 94i:35144

9. W. Magnus and S. Winkler, Hill's Equation, John Wiley and Sons, New York, 1966. MR 33:5991

10. P. Ungar, Stable Hill equations, Comm. Pure Appl. Math. 14 (1961), 707-710. MR 31:423

Department of Mathematics, National Tsing Hua University, Hsinchu, Taiwan 30043

E-mail address: mjhuang@math.nthu.edu.tw 